\title{
Critical long-range vector model in the UV
}

\author{
Soumangsu Chakraborty and Mikhail Goykhman \\ Department of Theoretical Physics, Tata Institute of Fundamental Research, \\ Homi Bhabha Road, Mumbai 400005, India \\ The Racah Institute of Physics, The Hebrew University of Jerusalem, \\ Sderot Magnes, Jerusalem 91904, Israel \\ E-mail: soumangsuchakraborty@gmail.com, \\ michael.goykhman@mail.huji.ac.il
}

ABSTRACT: We study interacting critical UV regime of the long-range $O(N)$ vector model with quartic coupling. Analyzing CFT data within the scope of $\epsilon$ - and $1 / N$-expansion, we collect evidence for the equivalence of this model and the critical IR limit of the cubic model coupled to a generalized free field $O(N)$ vector multiplet.

Keywords: Conformal Field Theory, Renormalization Group, 1/N Expansion

ARXIV EPRINT: 2108.10084 


\section{Contents}

1 Introduction and summary 1

$\begin{array}{lll}2 & \text { Anomalous dimensions and beta functions } & 7\end{array}$

$\begin{array}{lll}3 & \text { Fixed points } & 10\end{array}$

4 CFT data and critical duality 13

5 Discussion $\quad 14$

$\begin{array}{ll}\text { A Some useful identities } & 15\end{array}$

\section{Introduction and summary}

Spin systems with the long-range order, and their continuous version given by the longrange vector models, have a long history, starting with the earlier works of [1-4]. Such models are characterized by the positive-valued parameter $s$ that controls the power-law decay of the long-range spin interaction, or a bi-local kinetic term of the corresponding generalized free field. Dependence on the parameter $s$ is in particular inherited in the critical regime of these models, thereby substantially enriching their phase structure. In this paper we intend to explore the UV critical regime of the $d$-dimensional long-range $O(N)$ vector model with quartic interaction achieved within the domain $s<d / 2$.

The long-range $O(N)$ vector model is obtained by perturbing the generalized free field action for the scalar multiplet $\phi^{i}, i=1, \ldots, N$, by the local quartic interaction term [2],

$$
S=C(s) \int d^{d} x \int d^{d} y \frac{\phi^{i}(x) \phi^{i}(y)}{|x-y|^{d+s}}+\frac{g_{4}}{N} \int d^{d} x\left(\phi^{i} \phi^{i}\right)^{2} .
$$

Here the bi-local kinetic term prefactor

$$
C(s)=\frac{2^{s-1}}{\pi^{\frac{d}{2}}} \frac{\Gamma\left(\frac{d+s}{2}\right)}{\Gamma\left(-\frac{s}{2}\right)}
$$

was chosen so that the propagator of the field $\phi$ is canonically normalized in momentum space, ${ }^{1}$

$$
\left\langle\phi^{i}(p) \phi^{j}(q)\right\rangle=(2 \pi)^{d} \delta^{(d)}(p+q) \delta^{i j} \frac{1}{p^{s}} .
$$

In what follows, we will tend to skip explicitly keeping track of the $O(N)$ indices and the associated Kronecker symbols where it does not cause a confusion.

\footnotetext{
${ }^{1}$ See, e.g., [5] for a recent discussion.
} 
The scaling dimension of the field $\phi$ is fixed exactly by the bi-local kinetic term in the action (1.1) to be [2]

$$
\Delta_{\phi}=\frac{d-s}{2}
$$

Unitarity bound on $\Delta_{\phi}$ then restricts us to consider $s \leq 2$. The quartic coupling constant $g_{4}$ in the action (1.1) is relevant when $s>d / 2$. In this case the theory flows from the UV fixed point $g_{4}=0$ to a non-trivial critical regime in the IR. While this theory does not possess a local stress-energy tensor, a comprehensive argument has been provided to support the claim that its critical regime is described by an interacting CFT [5-16]. The bounds on $s$ impose the condition $d<4$ on the allowed space-time dimension, that accommodates a non-trivial critical regime in the IR. When $s=d / 2+\epsilon$, one can study the IR fixed point perturbatively in $\epsilon[2]$.

Besides being restricted from above by the unitarity bound $s \leq 2$, one can see that for large enough $s$ the long-range model $(1.1)$ will transition to a short-range regime $[17,18]$. In other words, the long-range IR CFT is found in the domain $d / 2<s<s_{\star}$. The upper bound $s_{\star}$ of the long-range domain can be determined by imposing the condition of relevance of the bi-local kinetic term. Indeed, consider the short-range $O(N)$ vector model describing dynamics of the field $\hat{\phi}$, with the local kinetic term $\frac{1}{2} \int d^{d} x(\partial \hat{\phi})^{2}$, perturbed by a quartic interaction $\left(\hat{\phi}^{2}\right)^{2}$. At the IR fixed point one obtains the scaling dimension $[\hat{\phi}]_{\mathrm{IR}}=\frac{d}{2}-1+\gamma_{\hat{\phi}}$. Evaluating the scaling dimension of the bi-local kinetic term for the field $\hat{\phi}$, one arrives at $s-s_{\star}$, where $s_{\star}=2-2 \gamma_{\hat{\phi}}$. The bi-local kinetic term therefore becomes more relevant when $s<s_{\star}$, while the point $s=s_{\star}$ defines the long-range-short-range crossover. CFT data is expected to be smooth across the crossover $[17,18]$.

The nature of the short-range CFT to the right of the crossover point, $s>s_{\star}$, was elucidated in $[11,13]$. It is given by the critical short-range $O(N)$ vector model plus a decoupled generalized free field $\chi$ of dimension $\Delta_{\chi}=(d+s) / 2$. On the other hand, when $d / 2<s<s_{\star}$, a coupling $\lambda \hat{\phi}^{i} \chi^{i}$ triggers an RG flow, that terminates at the long-range CFT describing the IR critical regime of the model $(1.1)[11,13]$. The RG flow triggered by the coupling $\lambda$ can be studied perturbatively in the vicinity of $s_{\star}$, complementing a perturbative study near the other edge, $s=d / 2$, of the long-range window, $d / 2<s<s_{\star}[11,13]$.

The CFT data of the model (1.1) was recently extensively studied in [5] in the large $N$ limit up to the next-to-leading order in $1 / N$ expansion, and for general values of $s$ and d. To this end, the Hubbard-Stratonovich formalism was used, that allows for the model to be formulated as

$$
S=C(s) \int d^{d} x \int d^{d} y \frac{\phi^{i}(x) \phi^{i}(y)}{|x-y|^{d+s}}+\int d^{d} x\left(-\frac{1}{4 g_{4}} \sigma^{2}+\frac{1}{\sqrt{N}} \sigma \phi^{2}\right)
$$


At the fixed point the scaling dimension of the Hubbard-Stratonovich field $\sigma$ is given by $[5,12,15]$

$$
\begin{aligned}
{[\sigma]_{\text {crit }}=} & s+\tilde{\gamma}_{\sigma}, \\
\tilde{\gamma}_{\sigma}= & -\frac{1}{N} \frac{4 \Gamma\left(\frac{s}{2}\right)^{2} \Gamma(d-s)}{\Gamma\left(\frac{d}{2}\right) \Gamma(s) \Gamma\left(d-\frac{3 s}{2}\right) \Gamma\left(\frac{d-s}{2}\right)^{3} \Gamma\left(s-\frac{d}{2}\right)^{2}}\left(\Gamma(s) \Gamma\left(d-\frac{3 s}{2}\right) \Gamma\left(\frac{d-s}{2}\right) \Gamma\left(s-\frac{d}{2}\right)\right. \\
& \left.-2 \Gamma\left(\frac{s}{2}\right) \Gamma\left(\frac{d}{2}-s\right) \Gamma(d-s) \Gamma\left(\frac{3 s}{2}-\frac{d}{2}\right)\right)+\mathcal{O}\left(\frac{1}{N^{2}}\right) .
\end{aligned}
$$

For $s>d / 2$, in the large $N$ limit, the $\sigma^{2}$ term in (1.5) is then irrelevant in the IR, while for $s<d / 2$ it is irrelevant in the UV, and has no influence on the corresponding CFT. Notice that near $s=d / 3$ we have

$$
\tilde{\gamma}_{\sigma}=\frac{1}{N} \frac{\kappa}{s-\frac{d}{3}}+\mathcal{O}\left(\left(s-\frac{d}{3}\right)^{0}\right)
$$

where we defined the positive-valued coefficient

$$
\kappa=\frac{16 \Gamma\left(\frac{d}{6}\right)^{4} \Gamma\left(\frac{2 d}{3}\right)^{2}}{3 \Gamma\left(-\frac{d}{6}\right)^{2} \Gamma\left(\frac{d}{3}\right)^{4} \Gamma\left(\frac{d}{2}\right)^{2}} .
$$

Therefore for $s<d / 3$ one can obtain arbitrarily large negative-valued $\tilde{\gamma}_{\sigma}$, resulting in the dimension $[\sigma]_{\text {crit }}$ dipping below the unitarity bound. Such a regime needs to be excluded.

To summarize, the model (1.5) achieves a non-trivial critical regime in the IR for

$$
d / 2<s<s_{\star}, \quad 2<d<4,
$$

and in the UV for

$$
d / 3<s<\min \left(d / 2, s_{\star}\right), \quad 2<d<6 .
$$

Notice that since at the leading order in $1 / N$ we have $s_{\star}=2+\mathcal{O}(1 / N)$, then in the large $N$ limit the upper bound in (1.11) is determined by whether we are working in low $(2<d<4)$ or higher $(4<d<6)$ dimensions.

The CFT data derived in [5] includes the ratio of the large- $N$ three-point functions amplitudes (OPE coefficients) ${ }^{2}$

$$
\left.\frac{\langle\sigma \sigma \sigma\rangle}{\langle\sigma \phi \phi\rangle}\right|_{\text {normalized }}=\frac{2^{d-2 s}(d-2 s) \sin \left(\frac{1}{2} \pi(d-2 s)\right) \Gamma\left(\frac{d}{2}-s\right)^{2} \Gamma\left(\frac{1}{2}(d-s+1)\right) \Gamma\left(\frac{1}{2}(3 s-d)\right)}{\pi \Gamma\left(\frac{s+1}{2}\right) \Gamma\left(d-\frac{3 s}{2}\right)},
$$

as well as the ratio of the next-to-leading to leading (in $1 / N$ expansion) OPE coefficient $\langle\sigma \phi \phi\rangle$

$$
\left.\delta C_{\phi \phi \sigma}\right|_{\text {normalized }}=\delta Z_{\phi \phi \sigma}+\delta \mathcal{U}_{\phi \phi \sigma}+A_{\phi}+\frac{A_{\sigma}}{2},
$$

where we refer the reader to [5] for details.

\footnotetext{
${ }^{2}$ The tag 'normalized' means that the propagators of the fields $\phi$ and $\sigma$ in position space have been normalized to unity.
} 
Our discussion above was centered around the goal to obtain a non-trivial fixed point in the IR. The corresponding arguments parallel those of the ordinary short-range (local) $O(N)$ vector model, that features a non-trivial IR criticality when $2<d<4$. When $d=4-\epsilon$, the corresponding critical regime of the short-range vector model admits a perturbative Wilson-Fisher description [19]. Pushing to higher dimensions, when $d=4+\epsilon$, the WF approach can be analytically continued, indicating existence of a fixed point in the UV rather than in the IR. This fixed point is achieved at negative values of the quartic coupling constant, $g_{4}$, and therefore the model is unstable. In fact, an interacting UV fixed point of the short-range $O(N)$ vector model is believed to exist in the entire range $4<d<6$, where the upper bound $d=6$ is due to the unitarity constraint on the scaling dimension of the Hubbard-Stratonovich field [20]. Given the problematic nature of this UV fixed point, it is desirable to get a better understanding of the corresponding CFT. This motivated [21] to argue for a critical duality between the UV fixed point of the higherdimensional $O(N)$ vector model and the IR fixed point of the certain cubic model. Such a duality (critical universality or, more specifically, a UV completion) has been successfully tested up to the fourth order in perturbation theory in $d=6-\epsilon$ dimensions, and in fact was argued to hold true in the entire range $4<d<6[22,23] .^{3}$

Similarly to the case of the short-range $O(N)$ vector model, described in the previous paragraph, the non-trivial criticality region $s>d / 2$ of the long-range vector model can be extended to $s<d / 2$ if we look for an interacting fixed point in the UV rather than in the IR. Indeed, when $s<d / 2$, the quartic interaction in the long-range model (1.1) is irrelevant, indicating an RG flow to an interacting regime in the UV. Together with the unitarity bound $s<2$ and the long-range bound $s<s_{\star}$ we obtain $s<\min \left(d / 2, s_{\star}\right){ }^{4}$ Analogously to the local short-range case, the instability issue is found yet again at the corresponding UV fixed point, as can be seen by a perturbative study at $s=d / 2-\epsilon$, rendering a negative fixed point value of the quartic coupling constant. The question is then whether one can construct a UV completion of the corresponding CFT in the spirit of [21]. This is the problem that we address in the current paper.

We propose that the UV fixed point of the model (1.1) within the range $0<s<d / 2$ is identical (critically dual) to the IR fixed point of the following model ${ }^{5}$

$$
S=C(s) \int d^{d} x \int d^{d} y \frac{\phi(x) \phi(y)}{|x-y|^{d+s}}+\int d^{d} x\left(\frac{1}{2}(\partial \sigma)^{2}+\frac{1}{2} g \sigma \phi^{2}+\frac{1}{6} h \sigma^{3}\right),
$$

describing dynamics of the vector multiplet of generalized free field $\phi^{i}, i=1, \ldots, N$, coupled to the singlet scalar $\sigma$ with the cubic self-interaction. Scaling dimensions of these fields are given by

$$
[\phi]=\Delta_{\phi}=\frac{d-s}{2}, \quad[\sigma]_{\mathrm{UV}}=\Delta_{\sigma}=\frac{d-2}{2} .
$$

\footnotetext{
${ }^{3}$ Formally one can consider the $O(N)$ vector model in the non-unitary domain $d>6$, where yet another model was argued in [24] to be critically equivalent to the $O(N)$ vector model.

${ }^{4}$ Recall that $\gamma_{\hat{\phi}}>0[22,25-28]$ and therefore $s_{\star}<2$.

${ }^{5}$ Since from now one we will be mostly discussing the model (1.14), we are going to recycle the symbols $S, \phi, \sigma$.
} 
Unitarity then demands $s<2$, which leads to $s<\min (d / 2,2)$. In fact, the model (1.14) transitions to the short-range regime when the $s>s_{\star}$, that can be determined by calculating scaling dimension of the bi-local kinetic term for the field $\tilde{\phi}$ in the critical cubic short-range model. Scaling dimension of the latter is equal to the scaling dimension of the field $\hat{\phi}$ of the short-range $O(N)$ vector model with quartic interaction [21]. Therefore at the outset we restrict to the range $0<s<\min \left(d / 2, s_{\star}\right)$.

Similarly to [21], we are looking for an IR stable fixed point on the $(g, h)$ plane. We will argue for the equivalence of the corresponding critical theory and the UV fixed point of the $O(N)$ vector model (1.1), by calculating and matching CFT data, such as scaling dimensions and OPE coefficients. ${ }^{6}$

Establishing the critical duality between the models (1.1), (1.14) begins with matching of the d.o.f. To that end, we use the Hubbard-Stratonovich representation (1.5) of the long-range vector model. The fields $\phi^{i}$ and $\sigma$ are accordingly matched in (1.5), (1.14). By construction, the scaling dimension $[\phi]=\Delta_{\phi}=(d-s) / 2$ is exact in interacting theory in both of these models. Below in this paper we will also match the CFT data of the cubic model (1.14) with its counterparts $(1.6),(1.7),(1.12),(1.13)$ in the long-range vector model (1.5).

We will study the model (1.14) in a perturbative regime in the couplings $g, h$, by choosing the specific values of the parameters $d, s$. Mass dimensions of the coupling constants $g, h$ in the action (1.14) are given by

$$
[g]_{\mathrm{UV}}=s+1-\frac{d}{2}=\epsilon_{2}, \quad[h]_{\mathrm{UV}}=3-\frac{d}{2}=\frac{\epsilon_{1}}{2},
$$

where we defined

$$
d=6-\epsilon_{1}, \quad s=\frac{d}{2}-1+\epsilon_{2}=2-\frac{\epsilon_{1}}{2}+\epsilon_{2} .
$$

Therefore, relevance of the couplings in the UV imposes the constraints

$$
d \leq 6, \quad s \geq d / 2-1,
$$

that lead to $\epsilon_{1,2} \geq 0$. Notice that the constraint on $s$ in (1.18) selects a sub-region $d / 2-1 \leq$ $s<\min \left(d / 2, s_{\star}\right)$ within the domain $s<\min \left(d / 2, s_{\star}\right)$, where the long-range $O(N)$ vector model flows to a non-trivial critical regime in the UV.

One can then perform Wilson-Fisher perturbative expansion in $\epsilon_{1,2}$. We will be working at the linear order $\mathcal{O}\left(\epsilon_{1,2}\right)$. It is convenient to denote

$$
\alpha=\frac{\epsilon_{2}}{\epsilon_{1}}<\frac{1}{2}+\mathcal{O}\left(\frac{1}{N}\right)
$$

where the last inequality follows from the bound $s<\min \left(d / 2, s_{\star}\right)=s_{\star}=2+\mathcal{O}(1 / N)$, taking into account $d=6-\epsilon_{1}$ and $\gamma_{\tilde{\phi}}=\mathcal{O}(1 / N)$. Our results will be exact to all orders in $1 / N$. Subsequently performing algebraic expansion to the next-to-leading order in $1 / N$ one can compare the CFT data that we derive in this paper with its counterpart in the

\footnotetext{
${ }^{6}$ Another example of a UV completion of non-local vector model was discussed in [15], that considered the case of $s=1$, corresponding to a boundary CFT.
} 
long-range vector model. The latter (having been derived in $1 / N$ expansion but for general $d$ and $s$ ) is in turn to be expanded to a linear order in $\epsilon_{1,2}$.

While specifics of the match of CFT data on both sides of the proposed critical duality will be carefully detailed below in this paper, we can briefly outline some important ingredients that already supply a promising indication that the duality is going to hold (at least within the $\epsilon$-expansion). The fixed point values for the couplings $g, h$, determined at the leading order in $\epsilon_{1,2}$, and subsequently expanded in $1 / N$, have the form

$$
\begin{aligned}
& g=g_{0}\left(1+\frac{g_{1}}{N}+\mathcal{O}\left(\frac{1}{N^{2}}\right)\right), \\
& h=h_{0}\left(1+\frac{h_{1}}{N}+\mathcal{O}\left(\frac{1}{N^{2}}\right)\right),
\end{aligned}
$$

where

$$
g_{0}, h_{0}=\mathcal{O}\left(\sqrt{\frac{\epsilon_{1}}{N}}\right) .
$$

One can see that the Callan-Symanzik equation for the $\langle\phi \phi \sigma\rangle$ three-point function then implies (here we take into account that $\left.\gamma_{\phi}=0\right)^{7}$

$$
\beta_{g}=-\epsilon_{2} g+g \gamma_{\sigma}+\mathcal{O}\left(\frac{1}{N^{3 / 2}}\right)
$$

and therefore at the non-trivial IR fixed point we obtain anomalous dimension

$$
\gamma_{\sigma}=\epsilon_{2}+\mathcal{O}\left(\frac{1}{N}\right)
$$

Consequently the IR scaling dimension of the field $\sigma$ is given by

$$
[\sigma]_{\mathrm{IR}}=\frac{d-2}{2}+\gamma_{\sigma}=s+\mathcal{O}\left(\frac{1}{N}\right) .
$$

Notice that unlike the case (1.7) of the long-range $O(N)$ vector model, the anomalous dimension $\gamma_{\sigma}$ has $\mathcal{O}\left(1 / N^{0}\right)$ component. Combined with the UV dimension (1.15) it results in the large- $N$ expression (1.25), that agrees with its counterpart in the critical long-range vector model (1.6).

Furthermore, we obtain the IR scaling dimension of the cubic coupling $[h]_{\mathrm{IR}}=d-3 s+$ $\mathcal{O}\left(\frac{1}{N}\right)$. The cubic term, whose presence in the model (1.14) naively distinguishes it from the critical $O(N)$ vector model (1.5), is then irrelevant in the IR, provided that $s>d / 3$. This bound is compatible with the unitarity bound $s \leq 2$, if we demand that $d \leq 6$. Such a restriction on space-time dimension is also compatible with our assumptions (1.18), that also makes the $s>d / 3$ constraint stronger than $s \geq d / 2-1$ constraint in (1.18). This means that the region that the model (1.14) allows us to study is ${ }^{8}$

$$
d / 3<s<\min \left(d / 2, s_{\star}\right),
$$

that agrees with (1.11). In terms of $\epsilon_{1,2}$ it gives $\alpha>1 / 6,{ }^{9}$ that in combination with (1.19)

\footnotetext{
${ }^{7}$ At the same time, the Callan-Symanzik equation for the $\langle\sigma \sigma \sigma\rangle$ three-point function at the leading order $\mathcal{O}\left(1 / N^{1 / 2}\right)$ includes a contribution from the counterterm $\delta h$. We consistently solve the Callan-Symanzik equations for both $\langle\phi \phi \sigma\rangle$ and $\langle\sigma \sigma \sigma\rangle$ in the main text.

${ }^{8}$ Similarly, one can see that the local kinetic term for $\sigma$ in (1.14) is irrelevant in the IR when $s>d / 2-1$, in agreement with (1.18).

${ }^{9}$ Indeed, using (1.17) we obtain $s-d / 3=\epsilon_{1}(\alpha-1 / 6)>0$, landing $s$ within the range (1.26).
} 
defines the domain

$$
\frac{1}{6}<\alpha<\frac{1}{2} .
$$

The rest of this paper is organized as follows. In section 2 we will carry out renormalization of the model (1.14) to linear order in perturbation theory, deriving anomalous dimension of $\sigma$ and the beta functions for the couplings $g, h$. We solve for the fixed points of this model in section 3 , and determine the IR stable fixed point on the $(g, h)$ plane. In section 4 we analyze the CFT data at the IR stable fixed point, and demonstrate that it matches the known CFT data at the UV fixed point of the $O(N)$ vector model. We discuss our results and possible future directions in section 5 . We assemble some well-known identities, useful for calculations in conformal perturbation theory in position space, in appendix A.

\section{Anomalous dimensions and beta functions}

We will perform our calculations in the model (1.14) perturbatively in the couplings $g, h$ near the free UV fixed point, working at the linear order in $\epsilon_{1,2}$. Free propagators of the fields $\phi, \sigma$ in position space are given by

$$
\langle\phi(x) \phi(0)\rangle=\frac{C_{\phi}}{|x|^{2 \Delta_{\phi}}}, \quad\langle\sigma(x) \sigma(0)\rangle=\frac{C_{\sigma}}{|x|^{2 \Delta_{\sigma}}},
$$

where the propagator amplitudes are given by

$$
C_{\phi}=\frac{1}{2^{s} \pi^{\frac{d}{2}}} \frac{\Gamma\left(\frac{d-s}{2}\right)}{\Gamma\left(\frac{s}{2}\right)}, \quad C_{\sigma}=\frac{\Gamma\left(\frac{d-2}{2}\right)}{4 \pi^{\frac{d}{2}}} .
$$

Interactions will introduce corrections to the propagator amplitudes, that are not essential for our goals. In fact, we will only be interested in the singular parts of the correlation functions that we compute. As we mentioned in the previous section, the scaling dimension $\Delta_{\phi}$ of $\phi$ will remain exact, while the scaling dimension $\Delta_{\sigma}$ of $\sigma$ will receive an anomalous contribution, that we intend to calculate.

We are going to employ perturbation theory in position space. The corresponding Feynman rules are given by
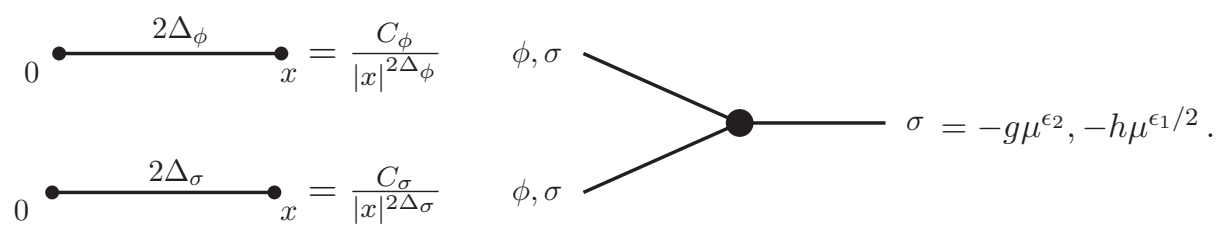

Here we have also introduced the dimensionless couplings $g, h$, taking into account the scaling dimensions (1.16), and defining an arbitrary RG scale $\mu$. (To eliminate clutter, we avoid using new notation for the dimensionless couplings.) A propagator line with a generic exponent will be assumed to have a unit amplitude

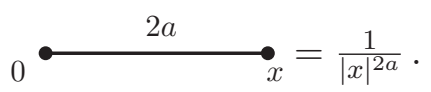


Diagrammatic calculations in position space can be performed using various well-known identities that we collected in appendix A.

The linear order, correction to the $\langle\phi \phi\rangle$ propagator is determined by the diagram

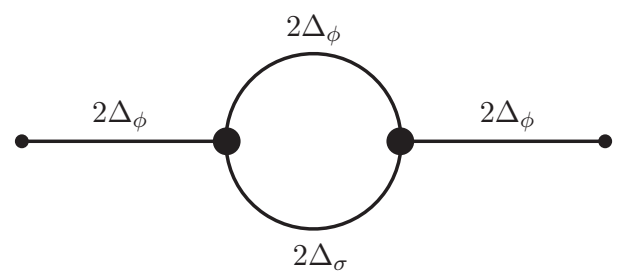

Combining this with the leading order propagator (2.1), we obtain

$$
\begin{aligned}
\langle\phi(x) \phi(0)\rangle & \supset \frac{C_{\phi}}{|x|^{d-s}}\left(1+g^{2}(\mu|x|)^{2 \epsilon_{2}} C_{\phi}^{2} C_{\sigma} V^{(\phi \phi)}\right), \\
V^{(\phi \phi)} & =U\left(\frac{d-s}{2}, d-1-\frac{s}{2}, 1+s-\frac{d}{2}\right) U\left(\frac{d-s}{2}, d-1-s, 1+\frac{3 s}{2}-\frac{d}{2}\right),
\end{aligned}
$$

where we took advantage of the simple behavior of loops in position space, discussed in appendix A, and used the propagator merging relation (A.1) and the function $U(a, b, c)$, given by (A.2). Expanding the second term in the parenthesis in (2.3) in $\epsilon_{2}$, we obtain a finite contribution, implying $\gamma_{\phi}=0$, in agreement with our expectation that scaling dimension of $\phi$ does not receive anomalous contributions.

Similarly, we have the following contributions to the $\langle\sigma \sigma\rangle$ propagator at the one-loop order in perturbation theory:

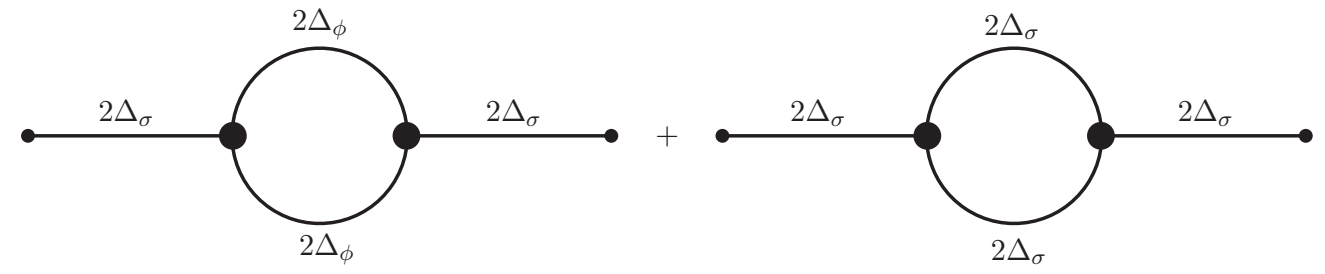

Together with the leading order propagator (2.1) we get

$$
\langle\sigma(x) \sigma(0)\rangle \supset \frac{C_{\sigma}}{|x|^{d-2}}\left(1+\frac{1}{2} C_{\sigma} C_{\phi}^{2} g^{2}(\mu|x|)^{2 \epsilon_{2}} V_{1}^{(\sigma \sigma)}+\frac{1}{2} C_{\sigma}^{3} h^{2}(\mu|x|)^{\epsilon_{1}} V_{2}^{(\sigma \sigma)}\right),
$$

where we denoted

$$
\begin{aligned}
& V_{1}^{(\sigma \sigma)}=U\left(\frac{d}{2}-1, d-s, s+1-\frac{d}{2}\right) U\left(\frac{d}{2}-1, d-s-1, s+2-\frac{d}{2}\right), \\
& V_{2}^{(\sigma \sigma)}=U\left(\frac{d}{2}-1, d-2,3-\frac{d}{2}\right) U\left(\frac{d}{2}-1, d-3,4-\frac{d}{2}\right) .
\end{aligned}
$$

Expanding the relative corrections in the parenthesis of (2.4) around $\epsilon_{2}=0$ and $\epsilon_{1}=0$ respectively, we obtain

$$
\begin{aligned}
\langle\sigma(x) \sigma(0)\rangle & \supset \frac{C_{\sigma}}{|x|^{d-2}}\left(1-2 \gamma_{\sigma} \log (\mu|x|)\right), \\
\gamma_{\sigma} & =\frac{h^{2}+N g^{2}}{12(4 \pi)^{3}}+\mathcal{O}\left(\epsilon_{1,2}^{2}\right),
\end{aligned}
$$


where we absorbed the pure divergence into the counterterm due to the wave-function renormalization of $\sigma$, that we have kept implicit.

We now proceed to the perturbative calculation of the three-point function $\langle\phi \phi \sigma\rangle$. At the leading order, it is given by a tree-level diagram, defined by the Feynman rule for the vertex corresponding to the coupling constant $g$. To get the three-point function $\langle\phi \phi \sigma\rangle$, we need to attach to this vertex two $\phi$ and one $\sigma$ propagator legs, and integrate over the location of the vertex insertion point. Using the uniqueness relation (A.4), we obtain

$$
\left\langle\phi\left(x_{1}\right) \phi\left(x_{2}\right) \sigma\left(x_{3}\right)\right\rangle=\frac{-\left.g \mu^{\epsilon_{2}} C_{\phi}^{2} C_{\sigma} U(2,2,2)\right|_{d=6}}{\left(\left|x_{12}\right|\left|x_{13}\right|\left|x_{23}\right|\right)^{2}} .
$$

At one-loop order, corrections are given by the following one-loop diagrams
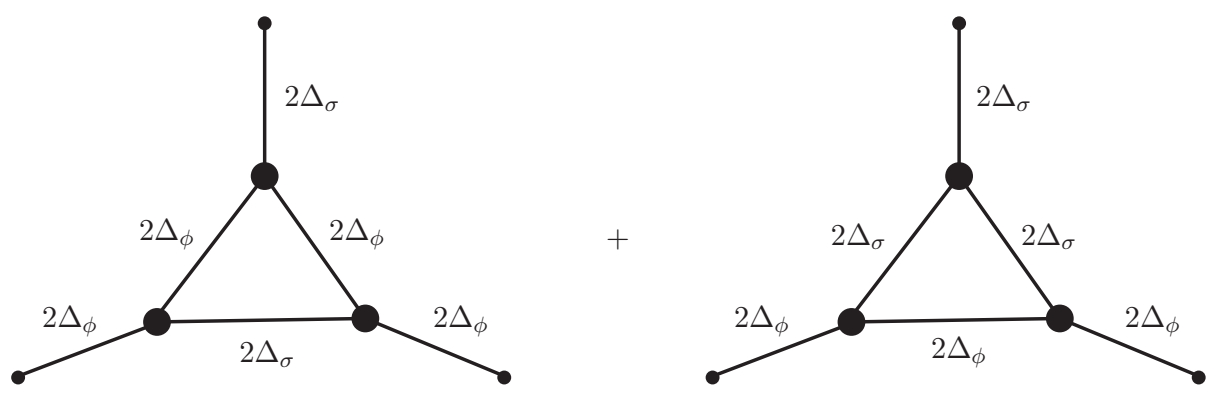

We are interested in the divergent structure of these diagrams, that can be extracted from integrating over the three internal vertices of the following graph

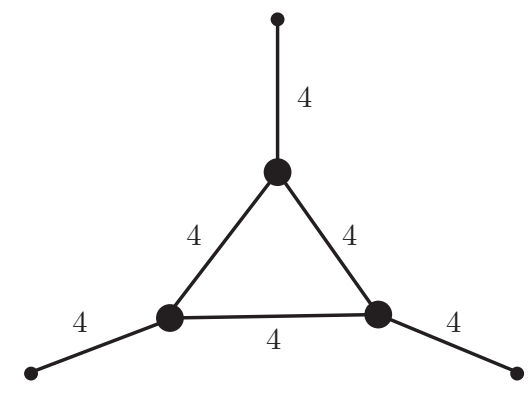

After one of the three vertices is integrated over, via the uniqueness relation (A.4), producing the factor of $\left.U(2,2,2)\right|_{d=6}=\pi^{3}$, we will encounter a logarithmically divergent integral, that we regulate using the UV cutoff $\mu_{0}$,

$$
\left.\int \frac{d^{6} x}{|x|^{6}}\right|_{|x|>1 / \mu_{0}}=\pi^{6} \log \left(\mu_{0}\right) .
$$

Such divergences are in fact removed with the $\delta g$ and $\delta h$ counterterms (that we keep mostly implicit in our calculation) proportional to $-\log \left(\mu_{0} / \mu\right)$, at the expense of introducing an arbitrary RG scale $\mu$. The final integral is taken using the uniqueness relation, producing another factor of $\left.U(2,2,2)\right|_{d=6}=\pi^{3}$. Assembling everything together, we obtain that the regularized graph $(2.9)$ is given by

$$
V^{(\phi \phi \sigma)}=\pi^{12} \log (\mu) .
$$


As a result, we obtain the one-loop corrections to $\langle\phi \phi \sigma\rangle$ given by

$$
\left\langle\phi\left(x_{1}\right) \phi\left(x_{2}\right) \sigma\left(x_{3}\right)\right\rangle \supset\left(-g^{3} C_{\phi}^{4} C_{\sigma}^{2} V^{(\phi \phi \sigma)}-g^{2} h C_{\phi}^{3} C_{\sigma}^{3} V^{(\phi \phi \sigma)}\right) \frac{1}{\left(\left|x_{12}\right|\left|x_{13}\right|\left|x_{23}\right|\right)^{2}} .
$$

Finally, extracting the $\mu$-dependent contribution obtained by dressing the $\sigma$ propagator of the tree level diagram (since $\phi$ does not have anomalous dimension, its propagator does not acquire a $\mu$-dependent dressing), we obtain

$$
\left\langle\phi\left(x_{1}\right) \phi\left(x_{2}\right) \sigma\left(x_{3}\right)\right\rangle \supset \frac{\left.2 g \gamma_{\sigma} C_{\phi}^{2} C_{\sigma} U(2,2,2)\right|_{d=6} \log (\mu)}{\left(\left|x_{12}\right|\left|x_{13}\right|\left|x_{23}\right|\right)^{2}} .
$$

Combining (2.8), (2.12), (2.13) and using the Callan-Symanzik equation ${ }^{10}$

$$
\left(\mu \frac{\partial}{\partial \mu}+\beta_{g} \frac{\partial}{\partial g}+\gamma_{\sigma}\right)\langle\phi \phi \sigma\rangle=0
$$

we obtain

$$
\beta_{g} \equiv \mu \frac{\partial}{\partial \mu} g=-\alpha \epsilon_{1} g+\frac{g}{12(4 \pi)^{3}}\left((N-12) g^{2}+h^{2}-12 g h\right)+\mathcal{O}\left(\epsilon_{1,2}^{2}\right) .
$$

Similarly, the three-point function $\langle\sigma \sigma \sigma\rangle$ at the tree level is given by

$$
\left\langle\sigma\left(x_{1}\right) \sigma\left(x_{2}\right) \sigma\left(x_{3}\right)\right\rangle=\frac{-\left.h \mu^{\epsilon_{1} / 2} C_{\sigma}^{3} U(2,2,2)\right|_{d=6}}{\left(\left|x_{12}\right|\left|x_{13} \| x_{23}\right|\right)^{2}},
$$

while the linear order corrections are obtained by dressing the $\sigma$ legs of the leading order tree graph, and including the one-loop vertex corrections
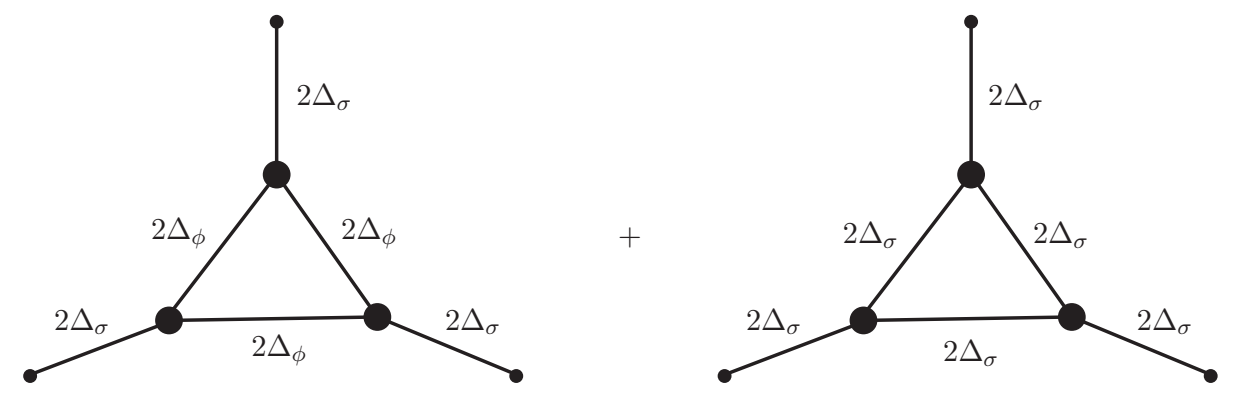

Carrying out the calculation, we arrive at

$$
\beta_{h} \equiv \mu \frac{\partial}{\partial \mu} h=-\frac{h \epsilon_{1}}{2}+\frac{1}{4(4 \pi)^{3}}\left(-3 h^{3}+N g^{2}(h-4 g)\right)+\mathcal{O}\left(\epsilon_{1,2}^{2}\right) .
$$

\section{$3 \quad$ Fixed points}

We now proceed to studying fixed points of the beta-functions $\beta_{g, h}$, given by $(2.15),(2.17)$. For general parameters $N, \alpha$, it is hard to find solutions to the equations $\beta_{g, h}=0$ analytically. To circumvent this difficulty, we will begin by considering large $N$ limit, and employ an algebraic $1 / N$ expansion, given by (1.20). Besides being a helpful analytic tool, in this case the algebraic $1 / N$ expansion will also conveniently allow us to compare the resulting CFT data with the CFT data derived by using the perturbative diagrammatic $1 / N$ expansion in the long-range $O(N)$ vector model (1.5).

\footnotetext{
${ }^{10}$ There is no contribution from $\beta_{h} \frac{\partial}{\partial h}$ at the considered order $\mathcal{O}\left(\epsilon_{1,2}^{3 / 2}\right)$.
} 

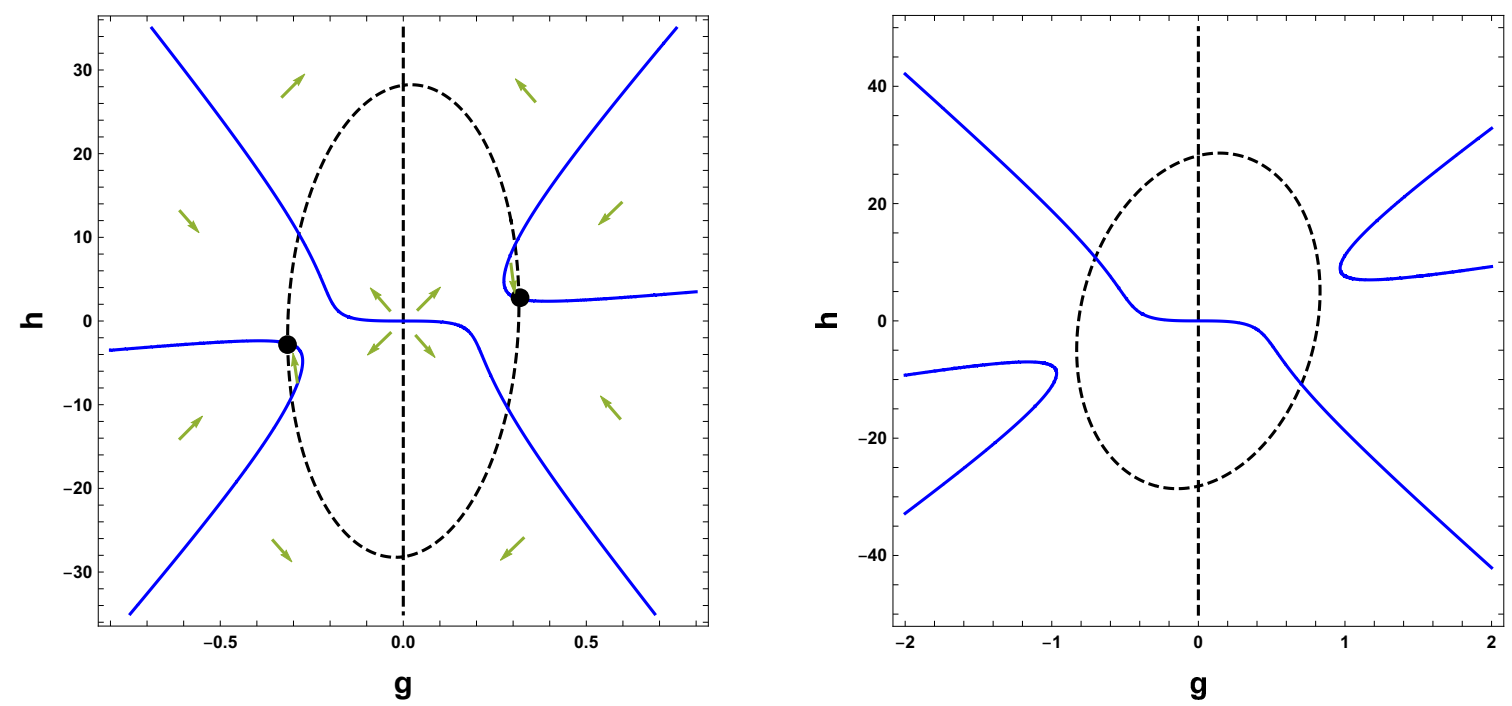

Figure 1. Contour plots for $\beta_{g}$ (black, dashed) and $\beta_{h}$ (blue, solid) for $\epsilon_{1}=0.1, \alpha=1 / 3, N=8000$ (left), $N=1200$ (right). RG flow direction (towards IR), determined by the signs of $\beta_{g, h}$ in various domains of the plot, are indicated with arrows. When $N=8000$ (left) the RG flow has a pair of IR stable fixed points on the $(g, h)$ plane (indicated with solid black dots). This fixed point for $\alpha=1 / 3$ disappears for $N \leq N_{\text {crit }}(\alpha=1 / 3)=2547$. In particular, it is absent for $N=1200$ (right).

The model (1.14) admits pairs of physically equivalent fixed points due to the $\mathbb{Z}_{2}$ symmetry,

$$
g \rightarrow-g, \quad h \rightarrow-h, \quad \sigma \rightarrow-\sigma .
$$

Carrying out $1 / N$ expansion of the $\beta_{g, h}=0$ equations, we obtain the following non-trivial solution $^{11}$

$$
\begin{aligned}
g_{0} & =16 \sqrt{\frac{3 \pi^{3} \alpha \epsilon_{1}}{N}}, & h_{0} & =\frac{24 \alpha}{6 \alpha-1} g_{0}, \\
g_{1} & =\frac{6\left(1-36 \alpha+132 \alpha^{2}\right)}{(6 \alpha-1)^{2}}, & h_{1} & =\frac{36 \alpha(19+6 \alpha(70 \alpha-17))-18}{(6 \alpha-1)^{3}} .
\end{aligned}
$$

The fixed point (3.2) is in fact paired up with a physically equivalent fixed point, obtained by the transformation $g_{0} \rightarrow-g_{0}, h_{0} \rightarrow-h_{0}$, due to the symmetry (3.1). Calculating determinant of the matrix of first derivatives of the beta functions for the solution (3.2), we get

$$
\mathcal{M} \equiv \operatorname{det}\left(\frac{\partial\left(\beta_{g}, \beta_{h}\right)}{\partial(g, h)}\right)=\alpha(6 \alpha-1) \epsilon_{1}^{2}+\mathcal{O}\left(\frac{1}{N}\right) .
$$

Therefore $\mathcal{M}>0$ in the region (1.27), indicating that the fixed point (3.2) is IR stable on the $(g, h)$ plane. The main statement of our paper is that the fixed point (3.2) is described by a CFT that is equivalent to the CFT in the UV critical regime of the non-local $O(N)$ vector model (1.5). While we support this claim by a perturbative calculation, we also suggest that such a duality holds true for the entire range (1.11).

\footnotetext{
${ }^{11}$ This procedure can be easily continued to higher orders in $1 / N$.
} 


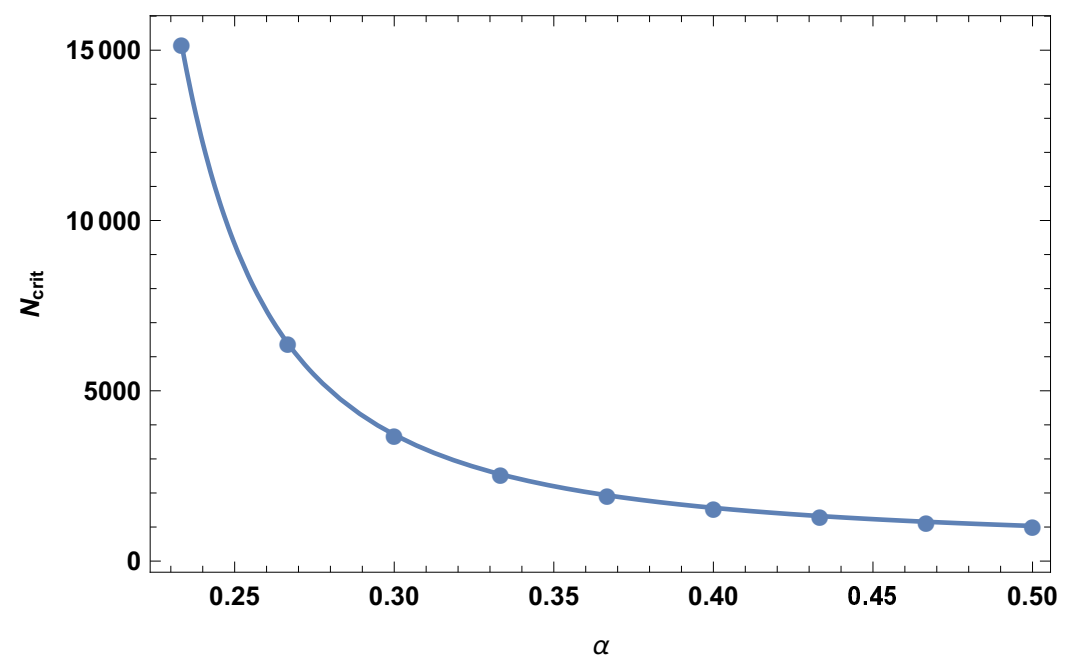

Figure 2. $N_{\text {crit }}$ as a function of $\alpha$. An IR stable fixed point on the $(g, h)$ plane exists only for large enough rank of the $O(N)$ group, $N \geq N_{\text {crit }}$.

Before proceeding with further study of the CFT at the fixed point (3.2), let us discuss behavior of all of the fixed points of the beta functions $\beta_{g, h}$ at various values of $N$. The system of two cubic equations $\beta_{g, h}=0$ can have up to nine different solutions. One of the solutions represents a free UV fixed point $g=h=0$, while eight other solutions are paired up due to the $\mathbb{Z}_{2}$ symmetry (3.1). One pair of solutions is given by $g=0$, $h= \pm 8 \pi^{3 / 2} i \sqrt{2 \epsilon_{1} / 3}$, and is not interesting for our purposes.

The remaining three pairs of solutions are accessible for general values of $\alpha$ and $N$ via numerics. We observe that for general $\alpha$ and small $N$, defined by $N<N_{\text {crit }}(\alpha)$, one has two complex-valued pairs of solutions, and a pair of real-valued solutions. The latter, however, are not IR stable on the $(g, h)$ plane, and therefore cannot be connected to the large- $N$ solution (3.2). However, for $N \geq N_{\text {crit }}(\alpha)$, one of the pairs of the complex-valued solutions turns real-valued and satisfies the requirement of the IR stability on the $(g, h)$ plane. We illustrate such a behavior in figure 1 , where we set $\epsilon_{1}=0.1, \alpha=1 / 3$, and consider the cases of $N=$ $8000, N=1200$. In this case, $N_{\text {crit }}(\alpha=1 / 3)=2548$. Correspondingly, one can see that the IR stable fixed point exists on the $N=8000$ plot, but disappears on the $N=1200$ plot.

The value $N_{\text {crit }}(\alpha)$ can be determined analytically as a function of $\alpha$. First, redefining $g=g_{0} x \sqrt{\epsilon_{1}}, h=h_{0} y \sqrt{\epsilon_{1}}$ we can rewrite $\beta_{g, h}=0$ equations as follows

$$
\begin{gathered}
(N-12) g_{0}^{2} x^{2}-12 g_{0} h_{0} x y+h_{0}^{2} y^{2}-768 \pi^{3} \alpha=0 \\
4 N g_{0}^{3} x^{3}-N g_{0}^{2} h_{0} x^{2} y+3 h_{0}^{3} y^{3}+128 \pi^{3} h_{0} y=0 .
\end{gathered}
$$

Written in such a form, this system of equations is in fact amenable to analytic solution. ${ }^{12}$ For our purposes it is sufficient to focus on the behavior of the square root term in one of the solutions, and determine the critical value of $N$ above which it becomes real-valued.

\footnotetext{
${ }^{12}$ This can be done in Mathematica.
} 
This yields

$$
\begin{aligned}
k_{1}= & \left(-(1-6 \alpha)^{6} \alpha(18 \alpha+1)^{2}(3 \alpha(324 \alpha(3 \alpha+1)+11)+2)^{3}(12 \alpha(\alpha(72 \alpha-11)+3)-1)^{\frac{1}{2}}\right. \\
k_{2}= & (18 \alpha(3 \alpha(4 \alpha(18 \alpha(12 \alpha(\alpha(972 \alpha(\alpha(54 \alpha(9 \alpha(108 \alpha(18 \alpha-1)+109)+43)+821)+93) \\
& \left.+7391)+381)+65)+215)-9)-5)+6 k_{1}-1\right)^{\frac{1}{3}} \\
N_{\text {crit }}= & \frac{12}{(6 \alpha-1)^{3}(18 \alpha+1)}\left(34992 \alpha^{4}-648 \alpha^{3}+684 \alpha^{2}+42 \alpha+k_{2}-2\right. \\
& \left.\quad+\frac{1}{k_{2}}\left(108 \alpha^{2}+1\right)(36 \alpha(3 \alpha(4 \alpha(27 \alpha+2)(108 \alpha(9 \alpha-1)+35)+3)-1)+1)\right) .
\end{aligned}
$$

Notice that $N_{\text {crit }}$ diverges when $\alpha \rightarrow 1 / 6$, i.e., at the lower end of the window (1.27). We plot it in figure 2, where we have also verified (3.5) by testing it numerically for various values of $\alpha$.

\section{CFT data and critical duality}

In section 3 we discussed the structure of perturbative fixed points of the couplings $g, h$ of the model (1.14) near $d=6$ and $s=d / 2-1$. We found numerically that for each $\alpha$ in (1.27) and large enough $N \geq N_{\text {crit }}(\alpha)$ there exists a real-valued fixed point that is IR stable on the $(g, h)$ plane. By expanding in $1 / N$ in the large $N$ limit, we arrived at an analytical solution (3.2) for the coupling constants $g, h$ at this fixed point.

While the fixed point (3.2) was determined perturbatively near $d=6$ and $s=d / 2-1$, we suggest that such an IR stable fixed point in fact exists for the range $d / 3<s<$ $\min \left(d / 2, s_{\star}\right)$, and that it is described by a CFT that is equivalent to the critical UV regime of the long-range $O(N)$ vector model (1.5). As we discussed in section 1, one can see that such a critical equivalence is indicated by the $1 / N$ structure (1.24) of the IR scaling dimension $[\sigma]_{\text {IR }}$ of the field $\sigma$, that can be easily determined from the Callan-Symanzik equation (1.23) in the large $N$ limit.

Furthermore, from (2.7), (3.2) one obtains

$$
[\sigma]_{\mathrm{IR}}=s+\frac{\epsilon_{1}}{N} \frac{12 \alpha(30 \alpha-1)}{6 \alpha-1}+\mathcal{O}\left(\epsilon_{1}^{2}, \frac{1}{N^{2}}\right) .
$$

On the other hand, expanding the anomalous dimension $\tilde{\gamma}_{\sigma}$ of the Hubbard-Stratonovich field (1.7) in the long-range $O(N)$ vector model in $\epsilon_{1}$ we obtain

$$
\tilde{\gamma}_{\sigma}=\frac{\epsilon_{1}}{N} \frac{12 \alpha(30 \alpha-1)}{6 \alpha-1}+\mathcal{O}\left(\epsilon_{1}^{2}, \frac{1}{N^{2}}\right)
$$

that is precisely in agreement with (4.1).

In a similar spirit, we can continue matching CFT data of the fixed point (3.2) of the model (1.14) with the critical long-range $O(N)$ vector model (1.5). One of the nontrivial consistency checks of the proposed critical duality involves matching of the relative next-to-leading over leading in $1 / N$ contributions to the OPE coefficient of the three-point 
function $\langle\phi \phi \sigma\rangle$. While at the fixed point (3.2) of the model (1.14) it is simply determined from the tree level diagram due to the $g$ interaction vertex,

$$
\left.\langle\phi \phi \sigma\rangle\right|_{\text {normalized }}=-\frac{\left.C_{\phi} C_{\sigma}^{\frac{1}{2}} U(2,2,2)\right|_{d=6} g_{0}\left(1+g_{1} / N+\mathcal{O}\left(\epsilon_{1}\right)\right)}{\left(\left|x_{12}\right|\left|x_{13}\right|\left|x_{23}\right|\right)^{2}} .
$$

in the long-range $O(N)$ vector model it was derived in [5] for general $d, s$, and is given by (1.13). Expanding the latter near $s=d / 2-1, d=6$, one obtains

$$
\left.\delta C_{\phi \phi \sigma}\right|_{\text {normalized }}=\frac{1}{N} \frac{6\left(1-36 \alpha+132 \alpha^{2}\right)}{(6 \alpha-1)^{2}}+\mathcal{O}\left(\epsilon_{1}, \frac{1}{N^{2}}\right),
$$

in agreement with $g_{1} / N$ due to $(4.3),(3.2) .{ }^{13}$

Continuing with the match of CFT data, we can perform $\epsilon_{1}$-expansion of the large- $N$ ratio of the normalized OPE coefficients $\langle\sigma \sigma \sigma\rangle /\langle\phi \phi \sigma\rangle$ in the model (1.5), given by (1.12), obtaining

$$
\left.\frac{\langle\sigma \sigma \sigma\rangle}{\langle\sigma \phi \phi\rangle}\right|_{\text {normalized }}=\frac{24 \alpha}{6 \alpha-1}+\mathcal{O}\left(\epsilon_{1}, \frac{1}{N}\right) .
$$

Its counterpart in the CFT data at the fixed point (3.2) of the model (1.14) is given by the ratio $h_{0} / g_{0}$, that it in fact agrees with.

\section{Discussion}

In this paper we discussed the UV critical regime of the long-range $O(N)$ vector model with quartic interaction (1.5). Working at the one-loop order in perturbation theory near $s=d / 2-1, d=6$, we provided evidence for the critical duality of this model and the IR fixed point of the cubic model coupled to a generalized free field (1.14). We suggest that such a critical universality in facts holds for the entire range of $d / 3<s<\min \left(d / 2, s_{\star}\right)$. Additional checks can be performed by matching more CFT data on both sides of the proposed duality, in particular by combining the techniques of $\epsilon$ - and $1 / N$-expansion, and studying higher orders thereof.

It would be interesting to see whether the model (1.14) at criticality can be obtained by deforming the critical cubic model of [21] by coupling it to a generalized free field $\chi$ of dimension $[\chi]=d-[\phi]=(d+s) / 2$, analogously to the case of the long-range $\phi^{4}$ model considered in $[11,13]$.

It was recently shown that critical long-range vector models play an important role in systems exhibiting the phenomenon of persistent symmetry breaking [30]. ${ }^{14}$ The cubic model proposed in this paper can shed additional light on properties of the corresponding CFTs.

\footnotetext{
${ }^{13}$ Importance of matching full CFT data, including sub-leading contributions to the OPE coefficients, in the context of testing critical duality of vector models was recently emphasized in [29].

${ }^{14}$ See $[31,32]$ for other recent work on persistent symmetry breaking in vector models.
} 


\section{Acknowledgments}

The work of S.C. is supported by the Infosys Endowment for the study of the Quantum Structure of Spacetime. The work of M.G. is partially supported by the Binational Science Foundation (grant No. 2016186), the Israeli Science Foundation Center of Excellence (grant No. 2289/18), and by the Quantum Universe I-CORE program of the Israel Planning and Budgeting Committee (grant No. 1937/12).

\section{A Some useful identities}

In this appendix we collect some known expressions and identities, that are useful to carry out perturbation theory calculations in position space.

Loop diagram in position space are simply additive:

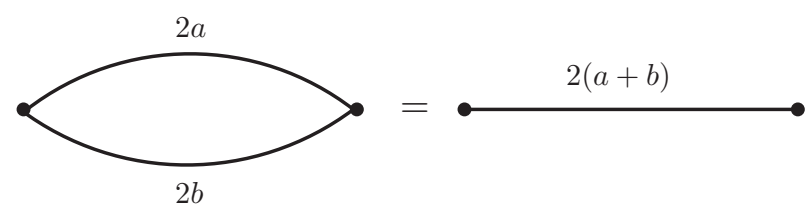

The propagator merging relation identity is given by

$$
\int d^{d} x_{2} \frac{1}{\left|x_{2}\right|^{2 a}\left|x_{1}-x_{2}\right|^{2 b}}=U(a, b, d-a-b) \frac{1}{\left|x_{1}\right|^{2 a+2 b-d}},
$$

where we defined

$$
U(a, b, c)=\pi^{\frac{d}{2}} A(a) A(b) A(c) .
$$

Here we have introduced

$$
A(x)=\frac{\Gamma\left(\frac{d}{2}-x\right)}{\Gamma(x)} .
$$

This relation can also be represented diagrammatically as

$$
\bullet 2 a \quad \bullet=\stackrel{2(a+b)-d}{\bullet} \bullet \times U(a, b, d-a-b)
$$

Uniqueness relation, valid for $a_{1}+a_{2}+a_{3}=d$, has the form [33, 34]

$$
\int d^{d} x \frac{1}{\left|x_{1}-x\right|^{2 a_{1}}\left|x_{2}-x\right|^{2 a_{2}}\left|x_{3}-x\right|^{2 a_{3}}}=\frac{U\left(a_{1}, a_{2}, a_{3}\right)}{\left|x_{12}\right|^{d-2 a_{3}}\left|x_{13}\right|^{d-2 a_{2}}\left|x_{23}\right|^{d-2 a_{1}}},
$$

and can be represented graphically as
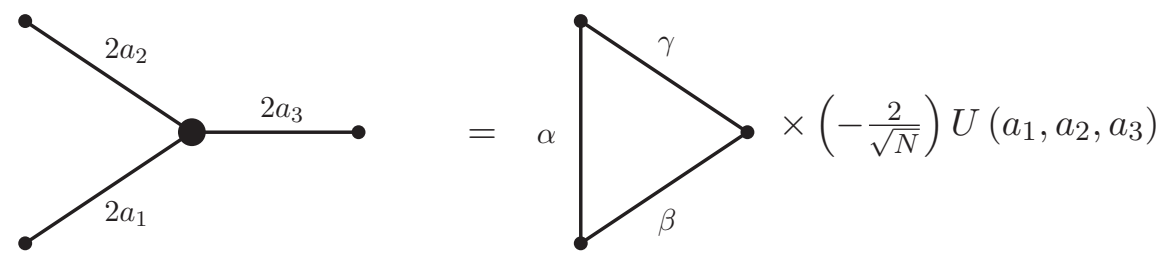

Here we have defined $\alpha=d-2 a_{3}, \beta=d-2 a_{2}, \gamma=d-2 a_{1}$. 
Open Access. This article is distributed under the terms of the Creative Commons Attribution License (CC-BY 4.0), which permits any use, distribution and reproduction in any medium, provided the original author(s) and source are credited.

\section{References}

[1] F.J. Dyson, Existence of a phase transition in a one-dimensional Ising ferromagnet, Commun. Math. Phys. 12 (1969) 91 [inSPIRE].

[2] M.E. Fisher, S.-K. Ma and B.G. Nickel, Critical exponents for long-range interactions, Phys. Rev. Lett. 29 (1972) 917 [InSPIRE].

[3] J.M. Kosterlitz, Phase transitions in long-range ferromagnetic chains, Phys. Rev. Lett. 37 (1976) 1577 [INSPIRE].

[4] M. Aizenman and R. Fernandez, Critical exponents for long-range interactions, Lett. Math. Phys. 16 (1988) 39.

[5] N. Chai, M. Goykhman and R. Sinha, Long-range vector models at large N, arXiv:2107.08052 [INSPIRE].

[6] D.C. Brydges, P.K. Mitter and B. Scoppola, Critical $\left(\Phi^{4}\right)_{3, \epsilon}$, Commun. Math. Phys. 240 (2003) 281 [hep-th/0206040] [INSPIRE].

[7] A. Abdesselam, A complete renormalization group trajectory between two fixed points, Commun. Math. Phys. 276 (2007) 727 [math-ph/0610018] [INSPIRE].

[8] G. Slade, Critical exponents for long-range $O(n)$ models below the upper critical dimension, Commun. Math. Phys. 358 (2018) 343 [arXiv:1611.06169] [INSPIRE].

[9] D. Benedetti, R. Gurau, S. Harribey and K. Suzuki, Long-range multi-scalar models at three loops, J. Phys. A $\mathbf{5 3}$ (2020) 445008 [arXiv:2007.04603] [INSPIRE].

[10] M.F. Paulos, S. Rychkov, B.C. van Rees and B. Zan, Conformal invariance in the long-range Ising model, Nucl. Phys. B 902 (2016) 246 [arXiv: 1509.00008] [INSPIRE].

[11] C. Behan, L. Rastelli, S. Rychkov and B. Zan, Long-range critical exponents near the short-range crossover, Phys. Rev. Lett. 118 (2017) 241601 [arXiv:1703.03430] [INSPIRE].

[12] S.S. Gubser, C. Jepsen, S. Parikh and B. Trundy, $O(N)$ and $O(N)$ and $O(N)$, JHEP 11 (2017) 107 [arXiv: 1703. 04202] [INSPIRE].

[13] C. Behan, L. Rastelli, S. Rychkov and B. Zan, A scaling theory for the long-range to short-range crossover and an infrared duality, J. Phys. A 50 (2017) 354002 [arXiv: 1703.05325] [INSPIRE].

[14] C. Behan, Bootstrapping the long-range Ising model in three dimensions, J. Phys. A 52 (2019) 075401 [arXiv:1810.07199] [InSPIRE].

[15] S. Giombi and H. Khanchandani, $O(N)$ models with boundary interactions and their long range generalizations, JHEP 08 (2020) 010 [arXiv: 1912.08169] [INSPIRE].

[16] E. Brezin, G. Parisi and F. Ricci-Tersenghi, The crossover region between long-range and short-range interactions for the critical exponents, Statist. Phys. 5 (2014) 010 [arXiv: 1407.3358].

[17] J. Sak, Recursion relations and fixed points for ferromagnets with long-range interactions, Phys. Rev. B 8 (1973) 281. 
[18] J. Sak, Low-temperature renormalization group for ferromagnets with long-range interactions, Phys. Rev. B 15 (1977) 4344.

[19] K.G. Wilson and M.E. Fisher, Critical exponents in 3.99 dimensions, Phys. Rev. Lett. 28 (1972) 240 [INSPIRE].

[20] G. Parisi, The theory of nonrenormalizable interactions. 1. The large $N$ expansion, Nucl. Phys. B 100 (1975) 368 [InSPIRE].

[21] L. Fei, S. Giombi and I.R. Klebanov, Critical $O(N)$ models in $6-\epsilon$ dimensions, Phys. Rev. D 90 (2014) 025018 [arXiv: 1404.1094] [INSPIRE].

[22] L. Fei, S. Giombi, I.R. Klebanov and G. Tarnopolsky, Three loop analysis of the critical $O(N)$ models in $6-\epsilon$ dimensions, Phys. Rev. D 91 (2015) 045011 [arXiv:1411.1099] [InSPIRE].

[23] J.A. Gracey, Four loop renormalization of $\phi^{3}$ theory in six dimensions, Phys. Rev. D 92 (2015) 025012 [arXiv: 1506.03357] [INSPIRE].

[24] J.A. Gracey, Six dimensional QCD at two loops, Phys. Rev. D 93 (2016) 025025 [arXiv: 1512.04443] [INSPIRE].

[25] A.C. Petkou, $C_{T}$ and $C_{J}$ up to next-to-leading order in $1 / N$ in the conformally invariant $0(N)$ vector model for $2<d<4$, Phys. Lett. B 359 (1995) 101 [hep-th/9506116] [INSPIRE].

[26] A. Petkou, Conserved currents, consistency relations and operator product expansions in the conformally invariant $O(N)$ vector model, Annals Phys. 249 (1996) 180 [hep-th/9410093] [INSPIRE].

[27] A.N. Vasiliev, Y.M. Pismak and Y.R. Khonkonen, Simple method of calculating the critical indices in the 1/N expansion, Theor. Math. Phys. 46 (1981) 104 [Teor. Mat. Fiz. 46 (1981) 157] [INSPIRE].

[28] A.N. Vasiliev, Y.M. Pismak and Y.R. Khonkonen, $1 / N$ expansion: calculation of the exponents $\eta$ and $\nu$ in the order $1 / N^{2}$ for arbitrary number of dimensions, Theor. Math. Phys. 47 (1981) 465 [Teor. Mat. Fiz. 47 (1981) 291] [INSPIRE].

[29] M. Goykhman and M. Smolkin, Vector model in various dimensions, Phys. Rev. D 102 (2020) 025003 [arXiv: 1911.08298] [INSPIRE].

[30] N. Chai, A. Dymarsky and M. Smolkin, A model of persistent breaking of discrete symmetry, arXiv:2106.09723 [INSPIRE].

[31] N. Chai, S. Chaudhuri, C. Choi, Z. Komargodski, E. Rabinovici and M. Smolkin, Thermal order in conformal theories, Phys. Rev. D 102 (2020) 065014 [arXiv:2005. 03676] [INSPIRE].

[32] N. Chai, E. Rabinovici, R. Sinha and M. Smolkin, The bi-conical vector model at $1 / N$, JHEP 05 (2021) 192 [arXiv:2011.06003] [INSPIRE].

[33] M. D'Eramo, G. Parisi and L. Peliti, Theoretical predictions for critical exponents at the $\lambda$-point of Bose liquids, Lett. Nuovo Cim. 2 (1971) 878 [INSPIRE].

[34] K. Symanzik, On calculations in conformal invariant field theories, Lett. Nuovo Cim. 3 (1972) 734 [INSPIRE]. 